\title{
Discordance of PIK3CA and TP53 Mutations Between Breast Cancer Brain Metastases and Matched Primary Tumors
}

\author{
ANNA THULIN \\ University of Gothenburg Institute of Clinical Sciences: Goteborgs universitet Institutionen for kliniska \\ vetenskaper
}

\section{Carola Andersson}

Kalmar County Hospital: Lanssjukhuset i Kalmar

\section{Elisabeth Rönnerman}

Sahlgrenska Sjukhuset: Sahlgrenska universitetssjukhuset

\section{Shahin De Lara}

Sahlgrenska Sjukhuset: Sahlgrenska universitetssjukhuset

\section{Chaido Chamalidou}

SkaS: Skaraborgs Sjukhus

\section{Arnd Schoenfeldt}

NU-sjukvården: NU-sjukvarden

\section{Anikó Kovács}

Sahlgrenska University Hospital: Sahlgrenska universitetssjukhuset

Henrik Fagman

University of Gothenburg: Goteborgs Universitet

Fredrik Enlund

Kalmar County Hospital: Lanssjukhuset i Kalmar

Barbro K. Linderholm ( $\nabla$ barbro.linderholm@oncology.gu.se)

Institution of Clinical Sciences https://orcid.org/0000-0003-1531-630X

\section{Research article}

Keywords: Breast cancer brain metastases, next generation sequencing (NGS), change of histological subtype PIK3CA, TP53

Posted Date: May 24th, 2021

DOl: https://doi.org/10.21203/rs.3.rs-525945/v1 
License: (c) (i) This work is licensed under a Creative Commons Attribution 4.0 International License. Read Full License 


\section{Abstract}

Purpose: Extensive data of mutations in breast cancer (BC) metastases has been published in recent years. However, these studies contain very few patients with brain metastasis (BM). Thus, there is limited knowledge of the biology of BCBM. We primarily aimed to compare the mutational and histological pattern of BM with matched primary breast cancer $(B C)$. Secondary aims were to determine mutations in BMs and PT in each BC subgroup (Luminal, HER2+ and TNBC) and survival according to changed or stable mutations between PTs and BMs.

Patients and methods: We investigated 57 BCBMs including 46 cases with the matched primary tumors (PT) by targeted Next Generation Sequencing (NGS) using the Cancer Hotspot Panel v2 (ThermoFisher Scientific) covering 207 targeted regions in 50 cancer related genes. BC subtype according to immunohistochemistry (estrogen- and progesterone receptors, HER2 and Ki67) was obtained by reevaluation of available tissue from BMs and PT.

Results: NGS results fulfilling sequencing quality criteria were obtained from $52 \mathrm{BM}(91.2 \%)$ and 41 (89.1\%) PT, out of which 37 were matched pairs. Pathogenic mutation was detected in $66 \%$ of PTs (27/41), and $62 \%$ of BMs (32/52). TP53 mutations were most frequent; $49 \%(20 / 41)$ of PTs and $48 \%$ $(25 / 52)$ in BMs, followed by PIK3CA mutations; 22\% (9/42) in PTs and 25\% (13/52) in BMs. Mutations in $C D H 1, E G F R, H R A S, R B 1 C D K N 2 A$ and PTEN were detected in single pairs or single samples. Mutational pattern was discordant in $24 \%$ of matched pairs. Standard BC markers was discordant in $26 \%$, with a loss of the estrogen receptor and a change from Luminal A to other subtypes as the most common. Changes of mutations in BMs compared with PT did not influence survival after diagnose of BM $(p=0.4395)$.

Conclusions: We show a discordance of PIK3CA and TP53 mutations, as well as IHC BC subgroups in $25 \%$ of the matched pairs of BM and PT, which confirms the need for re-evaluation of mutations, as well as standard BC markers by immunohistochemistry in the BM. Since there are difficulties in obtaining tissue from BM, analysis of cell-free DNA from cerebrospinal fluid (CSF) may be a way forward.

\section{Introduction}

Despite increasingly effective treatment, about $20 \%$ of patients with primary breast cancer (BC) suffer from metastatic disease and $15-40 \%$ of these patients eventually develop brain metastases (BM) [1]. Due to limited treatment options, and debilitating symptoms that greatly affect quality of life, BM is a dreaded outcome [2]. There is a large variation in survival after diagnosis of BM that ranges from Triple Negative Breast Cancer (TNBC), with a median survival of 4-5 months, to Human Epidermal growth factor Receptor 2 (HER2) positive (HER2+) BC with a median survival following BM diagnosis of 9-16 months $[3,4]$. The subtype of BC influences the risk of developing BMs and the BM free interval, with significantly higher incidence and shorter interval from recurrence to diagnosis of BM for patients with TNBC and HER2 + as compared to luminal tumors $[5,6]$. 
Local treatment such as surgery or radiotherapy forms the basis of BM treatment. There is also a clear evidence of systemic therapies having an effect on BM in patients ineligible for local treatment $[7,8]$. However, the blood-brain-barrier as well as the blood-tumor-barrier hinders the passage of systemic therapies to the central nervous system, hampering the effect of systemic treatment on BMs [9]. Another clinical problem is the difficulty to access metastatic tissue from the brain. Repeated studies have shown a discordance in the expression of steroid receptor and HER2 between metastatic lesions and the primary $\mathrm{BC}$ [10]. Thus, characterization of metastatic lesions is crucial for correct treatment decisions. The introduction of efficient therapies with targets like HER2, CDK4/6, PARP, and PIK3CA are being developed. Hence, the need for analysis of $\mathrm{BC}$ tissue in the metastatic setting is becoming apparent.

$\mathrm{BM}$ have become increasingly common in $\mathrm{BC}[6,11]$ and consequently, there is need for adequate information regarding the evolution of mutations, and histology in the metastatic process for correct therapy decisions [12-14]. Do targetable mutations in BMs differ compared to that of the previous primary tumor (PT)? There are studies investigating this topic in metastatic lesions [15]. However, due to difficulties to sample tissue, BMs represent very few of investigated metastatic lesions.

We primarily aimed to compare the histology and mutational pattern of BMs with matched primary BC using next generation sequencing (NGS) and immunohistochemistry (IHC). Secondly, we aimed to investigate the mutational patterns in each BC subgroup (Luminal, HER2 + and TNBC), and survival according to changed or stable mutations between PTs and BMs.

\section{Material And Methods}

\section{Patients}

From hospital records of all diagnostic codes, patients with BMs from BC between 1994 and 2014 were identified. Patients received treatment of the primary BC in any of four hospitals in the western region of Sweden. The diagnoses were confirmed in the patient's charts and patients with available material from BMs were selected. Clinical characteristics, type of metastasis, progression and survival were extracted from patient charts. PTs and BM tissue were evaluated, as specified below, when sufficient material was available. Data from the original report was utilized if deemed appropriated by the responsible breast pathologist if there was insufficient material available for re-evaluation by IHC. The study was conducted in accordance with the Declaration of Helsinki and the Sahlgrenska University Hospital Ethical Review Board; Gothenburg, Sweden approved the study (460-09, T592-14). Approval for the chart review and bio bank extractions was granted from each head of the participating departments.

\section{Immunohistochemistry (IHC)}

The available material was re-evaluated for histological type, nuclear grade, and receptor status. ER/PR, Ki67 and HER2 (HercepTest) immunohistochemistry was performed as per standard procedures using the Dako Autostainer Link and the EnVision ${ }^{\text {TM }}$ FLEX detection systems according to the manufacturer's 
instructions. HercepTest was followed by SISH when the IHC was judged as $2+$ or $3+$. For a more detailed description, see a previous publication. [6].

\section{Next Generation Sequencing}

\section{Preparation of sample library and next generation sequencing (NGS)}

DNA isolation from FFPE sections containing a minimum of $25 \%$ neoplastic cells as assessd by breast pathologists was performed using the QIAamp DNA FFPE tissue kit (Qiagen Gmbh, Hilden, Germany). DNA concentration was determined using NanoDrop ${ }^{\text {TM }} 3300$ with Quant-IT Picogreen dsDNA assay kit (ThermoFisher Scientific, Waltham, MA, USA). Ten ng of DNA was used to prepare barcoded libraries with the Ion AmpliSeq ${ }^{\text {TM }}$ Library kit 2.0 (ThermoFisher). The Cancer Hotspot Panel v2 (ThermoFisher Scientific) covering 207 targeted regions in 50 cancer related genes was used (https://tools.lifetechnologies.com/content/sfs/brochures/lon-AmpliSeq-Cancer-Hotspot-Panel-Flyer.pdf). Template preparation and enrichment was performed with the lonChef ${ }^{\mathrm{TM}}$ Instrument (ThermoFisher Scientific). Eight barcoded samples were pooled per lon $318^{\mathrm{TM}} \mathrm{v} 2 \mathrm{BC}$ chip and sequenced on the lon $\mathrm{PGM}^{\mathrm{TM}}$ System (ThermoFisher Scientific). All steps were performed according to the manufacturer's instruction. Matched germline DNA from the patients was not available in this retrospective investigation.

\section{Data processing}

After alignment to the hg19 human reference genome, variant calling by the Torrent Suite Software v4.2.1.0 and filtering of described SNPs was performed. Variants were visually inspected with the Integrative Genomics Viewer (IGV; Broad Institute, Cambridge, MA, USA). Mutations were manually curated as pathogenic, likely pathogenic, variants of unknown significance or as benign. For ten samples, NGS results could not be obtained due to insufficient quality of DNA. For individual variants the minimum accepted read depth was 500 and if the read depth was $<1000$, an allele frequency of $>5 \%$ was required for positive variant calling.

\section{Statistical Methods}

Tests of differences between subgroups and mutations was performed using Chi-square test or Fischer Exact test, depending on the number of expected values, with $5 \%$ significance as the limit to reject the null hypothesis. Brain Metastasis Specific Survival (BMSS) i.e. time from diagnosis of BM to death were estimated by the Kaplan Meier estimator, and compared by the log rank test. Two patients who had not undergone surgery were removed from survival analyses. IBM SPSS Statistics $25^{\mathrm{TM}}$ was used for statistical calculations.

\section{Results}

A total of 69 patients who had undergone surgery of breast cancer BM were retrieved from chart studies and by diagnostic codes. For 46 of these, archived tissue of the BMs as well as from the PT were 
available. For 11 patients, only the BM but not the PT was available. A total of 57 BMs and 46 PTs were thus included in the study. Of 57 patients with BM, 55 had undergone surgery (96\%). The median age at time of BM diagnosis was $53(29-75)$ years. The 22 patients with extra-cranial disease (ED) prior to BM had a median time from ED to BM of 18.1 months (range 1.4-92 months). For detailed data on clinical characteristics and survival at the time of diagnose of BM, please refer to Tables 1-3.

NGS results fulfilling sequencing quality criteria were obtained for 37 of the 46 matched PT/BM pairs and from 10 of the 11 unmatched BMs. For nine matched pairs, sequencing results were obtained for either only the PT $(n=4)$ or the BM $(n=5)$. In total, NGS results from 41 PTs and 52 BMs including the 37 matched PT/BM pairs were obtained (Fig. 1). For IHC subtype

\section{Genetic profile of PT and BM}

Twenty-seven PTs (66\%) and 32 BMs (62\%) exhibited at least one pathogenic mutation (Fig. 2). A pathogenic JAK2 p.V617F mutation in a patient with previously diagnosed myeloproliferative disease was excluded from further analysis. The most commonly mutated gene in the dataset was TP53, with a mutation frequency of $44 \%(20 / 42)$ in PTs and 44\% (25/52) in BMs. PIK3CA mutations were the second most prevalent, with a mutation frequency of $20 \%(9 / 42)$ in PTs and $23 \%(13 / 52)$ in BMs. Of note, a $C D H 1$ mutation was present in a PT/BM pair of lobular BC. Moreover, non-recurrent mutations in EGFR, HRAS and RB1 were detected in single PT/BM pairs as well as mutations in CDKN2A and PTEN in single samples of PT or BM.

\section{Mutational findings in matched pairs of BM and PT}

In the matched pairs, similarly, the most prevalent mutation was in TP53 in 43\% (16/37) of the matched pairs (Table 5). Mutated TP53 was found in three Luminal PTs and four paired BMs (27\%/36\%), eight TNBC PTs and ten paired BMs (47\%/59\%), and six HER2 positive PTs and five paired BMs $(67 \% / 55 \%)$. Mutations in PIK3CA was the second most prevalent mutation in this material. In matched PT/BM pairs, PIK3CA mutations were found in two PTs and four BMs of Luminal B $(18 \% / 36 \%)$; three PTs and BMs in TNBC $(18 \% / 18 \%)$, and in one matched case of HER2 positive tumors $(11 \%)$. The numerical differences of TP53 and PIK3CA mutations in BC subgroups did not reach statistical significance $(p=0.228)(p=0.552)$ respectively.

\section{Mutational concordance in matched material}

The mutational profile of the genes present in the targeted panel was discordant between the PTs and BMs in nine cases out of 37 (24\%). The mutational differences displayed no discernible pattern. There were three cases of mutations present only in the PT, four with only mutations found in the BM, and one case of mutation in the same gene, but at another position BM. Of note, in two PT/BM pairs, a pathogenic PIK3CA mutation and five TP53 mutations were detected only in the BM. An altered mutational status did not affect survival; patients with a mutational change versus stable mutational pattern had a similar brain metastasis specific survival (BMSS) $(p=0.4395)$. 


\section{Immunohistochemical subtype in matched material}

IHC subtype was available for 46 matched PT and BM. Receptor status by IHC was discordant in 12 out of $46(26.1 \%)$ of the matched pairs (Fig. 3). The corresponding discordance rate between PT and BM for each BC subtype were as follows; Luminal A 100\% (6/6); Luminal B 0\% (0), TNBC 9\% (2/ 23), HER2+/ ER $+50 \%(3 / 6)$, and HER2+/ ER 20\% (1/5). The most common change in BC subtype was from Luminal A to Luminal B.

\section{Survival parameters according subtype}

Patients with Luminal and HER2+/ER + BC had the longest BMSS with a median of 28 months (6-64) and 34 months (7-62) respectively, whilst patients with HER2+/ER- and TNBC had BMSS of 11 months $(0-46)$ and eight months $(0-46)$ respectively $(p=0.0851)$. We found equal BMSS when patients with mutations in PIK3CA were compared to patients without PIK3CA mutation $(p=0.467)$. There was no difference in BMSS when patients with a TP53 mutation were compared with patients without documented TP53 mutation $(p=0.456)$.

\section{Discussion}

Difficulties in accessing intracranial tissue samples have hampered development of systemic treatments that could have an effect in the treatment of patients with BMs. To our knowledge, this is one of the largest cohorts of genetically characterized BC BM, consisting of 52 analyzed cases, most notably including 37 cases with matched PT, all from one region in Sweden. Despite this, the relatively small number of patients in each BC subgroup is a limitation of the present study, reflecting the difficulty to obtain matched PT/BM tissue. Sequencing results by NGS were successfully obtained in $90 \%$ of the BMs. At least one mutation, among 50 cancer driver genes, was present in $62 \%$ of the analyzed samples similar to previously published data [16-21]. Concordant with these studies TP53 and PIK3CA are the most commonly mutated driver genes, both in the BMs and PTs. Of these two mutations, PIK3CA are considered targetable, whilst TP53 mutations can be indirectly attacked through restoration of the transcriptional activity resulting in a functional wild-type TP53 protein [22]

The drug alpelisib, is a PI3Ka-specific inhibitor available for patients with recurrent PIK3CA mutated Luminal/HER2 negative BC [23]. Previous published data show that approximately $40 \%$ of ER + PT harbor a PIK3CA mutation [24]. Two studies that compared PIK3CA mutations in PTs and BC metastases reported mutations in $33 \%$ of PTs and $30 \%$ of metastases and $45 \%$ of PTs and $53 \%$ in metastases respectively $[18,25]$. There were few or no patients with BMs in the above mentioned studies. We report lower figures with PIK3CA mutations in breast cancer among patients with BMs with a mutation frequency of $20 \%$ in PTs and $23 \%$ in the BMs. Interestingly, a recently published systematic review investigated $164 \mathrm{BMs}$ with its matched PT in 126 patients extracted from 13 studies, found PIK3CA mutations in $22 \%$ of the patients with BMs [26]. These similarities raise the hypothesis that PIK3CA mutations is lower in BMs compared to other metastatic sites, however, additional studies are required to adequately answer this question. As expected, we found the highest proportion of PIK3CA mutations in 
Luminal BC. Of note, in our material two out of eight pathogenic PIK3CA mutations and five TP53 mutations were found in the BMs only and not in the PT underlining the need for re-evaluation of metastatic tissue or possibly by analysis of cell free DNA (cfDNA).

We found PIK3CA mutations as the second most common mutation after TP53 in matched pairs of TNBC with $18 \%$ PIK3CA mutations all concordant in PTs and BMs. This is in line with previous published data in which PIK3CA mutations was the second most common mutation after TP53, especially in basallike and luminal androgen receptor subtypes of TNBC [27-29]. The combined treatment of alpelisib and nab-paclitaxel is currently under investigation in pre-treated TNBC with either loss of PTEN expression or a PIK3CA mutation (NCT04251533).

The prognostic role of PIK3CA mutations in HER2 + BC has been extensively investigated with less effect of HER2 blocking therapy, both in the neo-adjuvant setting, and for recurrent $\mathrm{BC}$ in terms of lower $\mathrm{pCR}$ rates and shorter survival respectively $[30,31]$. We found PIK3CA mutations in only $10 \%$ of the HER $2+$ cases. This is lower than previously reported of $20-40 \%$. [24]. The limited number of HER2 + patients in our cohort may be the cause of this discrepancy. The effect of alpelisib in HER2 + breast cancer is under investigation in one ongoing and one completed clinical trial (NCT02038010; NCT04208178).

Almost $50 \%$ of the PTs in the present study population with a high proportion of TNBC harbored a TP53 mutation, in contrast to the $20-35 \%$ mutation prevalence reported in unselected primary BC [32]. The Cancer Genome Atlas reveals an enrichment of TP53 mutations in basal-like and HER2 enriched BC [24]. Interestingly, previous data reveal that a high proportion of patients with a TP53 mutation in the primary $B C$ developed BM $[33,34]$. We lack data on the molecular subtypes in our material, but find an increased number of TP53 mutations in TNBC and HER2/ER- BC. Enrichment of TP53 mutations in BMs was not seen in the HER2 amplified subgroup, a finding that must be interpreted with caution due to the very small sample size of the HER2 group.

Roughly $25 \%$ of the matched pairs changed IHC based BC subtype in the BMs. The most common change was from Luminal A in PT to other subtypes, in most cases Luminal B in BMs. This is in accordance with previous results in which 219 patients showed a $36 \%$ overall discordance with the most common change in form of loss of PgR [35]. In a review pooling a total of 3384 matched pairs of BC and metastases from all organs, BM showed a discordant median rate of $22 \%$ compared to $45 \%$ in liver metastases and $16 \%$ in lymph node metastasis [36]. TNBC was the most stable group with less than $10 \%$ showing a gain in ER (one case) and HER2 (one case). Other detected mutations in our series, $C D H 1$, EGFR, HRAS, RB1, CDKN2A and PTEN were rare, in general found in single or both samples from one patient.

A limitation of our current study is that genetic profiling was performed using a relatively small NGS panel of 50 genes. Even though the panel gives broad coverage of important cancer driver genes, more comprehensive sequencing might have provided further details in the landscape of mutation discordancy between PTs and BMs. Still, we believe that the relatively large number of matched PT and BM may 
increase knowledge about the biology of BMs, how the metastatic process affects actionable genes, ultimately helping patients suffering from BM.

\section{Conclusions}

In conclusion, we confirm mutations in TP53 and PIK3CA to be common in both primary breast tumors and BMs but the proportion varied depending on the subgroup. Mutation pattern, as well as IHC based subtypes were discordant in approximately $25 \%$ of the patients underlining the need for re-biopsy at disease progression. In this context, analysis of mutations in liquid biopsies by use of cell-free DNA (cfDNA) from cerebrospinal fluid has shown promising results [37-39].

\section{Declarations}

Ethical Approval: The study has been conducted in accordance with the Declaration of Helsinki and the Sahlgrenska University Hospital Ethical Review Board, Gothenburg, Sweden approved the study (460-09, T592-14). The need for informed consent was waived under the approval of the Ethical Review Board due to the retrospective design.

Consent for publication: Not applicable

Availability of data and materials: All pathological and uncertain mutations are added as a supplemental table. Clinical data is not included as this would allow for possibly identifying individuals.

Competing interests: The authors declare no conflicts of interest.

Funding: This study was supported by grants from the Swedish Breast Cancer Association (Bröstcancerförbundet), the Swedish State under the LUA-agreement (Sahlgrenska University Hospital, Gothenburg) (VGFOUREG-667831).

Author Contributions: BL, AT, and FE conceived of the study. CA, SDL, AK, ER, FE, and BL developed the methodology for the study. CA, ER, SHD, CC, AS, AK, FE and AT participated in data acquisition. AT, FE, HF, and $B L$ participated in data analysis and interpretation. AT, BL, and HF wrote and/ or edited the manuscript. All authors read and approved the final manuscript.

\section{References}

1. Lin NU, Bellon JR, Winer EP. CNS metastases in breast cancer. J Clin Oncol. 2004;22(17):3608-17.

2. Witzel I, et al. Breast cancer brain metastases: biology and new clinical perspectives. Breast Cancer Res. 2016;18(1):8.

3. Niikura $\mathrm{N}$, et al. Treatment outcomes and prognostic factors for patients with brain metastases from breast cancer of each subtype: a multicenter retrospective analysis. Breast Cancer Res Treat. 2014;147(1):103-12. 
4. Witzel I, et al. Treatment and outcomes of patients in the Brain Metastases in Breast Cancer Network Registry. Eur J Cancer. 2018;102:1-9.

5. Berghoff A, et al. Brain metastases free survival differs between breast cancer subtypes. Br J Cancer. 2012;106(3):440-6.

6. Thulin A, et al. Clinical outcome of patients with brain metastases from breast cancer - A population based study over 21 years. Breast. 2020;50:113-24.

7. Bartsch $\mathrm{R}$, et al. Impact of anti-HER2 therapy on overall survival in HER2-overexpressing breast cancer patients with brain metastases. Br J Cancer. 2012;106(1):25-31.

8. Brufsky AM, et al. Central nervous system metastases in patients with HER2-positive metastatic breast cancer: incidence, treatment, and survival in patients from registHER. Clin Cancer Res. 2011;17(14):4834-43.

9. Gerstner ER, Fine RL. Increased permeability of the blood-brain barrier to chemotherapy in metastatic brain tumors: establishing a treatment paradigm. J Clin Oncol. 2007;25(16):2306-12.

10. Yeung $\mathrm{C}$, et al. Estrogen, progesterone, and HER2/neu receptor discordance between primary and metastatic breast tumours-a review. Cancer Metastasis Rev. 2016;35(3):427-37.

11. Cagney DN, et al. Incidence and prognosis of patients with brain metastases at diagnosis of systemic malignancy: a population-based study. Neuro Oncol. 2017;19(11):1511-21.

12. del Rivero J, Kohn EC. PARP Inhibitors: The Cornerstone of DNA Repair-Targeted Therapies. Oncology (Williston Park). 2017;31(4):265-73.

13. Murthy R, et al. Tucatinib with capecitabine and trastuzumab in advanced HER2-positive metastatic breast cancer with and without brain metastases: a non-randomised, open-label, phase $1 \mathrm{~b}$ study. Lancet Oncol. 2018;19(7):880-8.

14. Andre F, et al. Alpelisib for PIK3CA-Mutated, Hormone Receptor-Positive Advanced Breast Cancer. N Engl J Med. 2019;380(20):1929-40.

15. Bertucci F, et al. Comparative genomic analysis of primary tumors and metastases in breast cancer. Oncotarget. 2016;7(19):27208-19.

16. Muller KE, et al. Targeted next-generation sequencing detects a high frequency of potentially actionable mutations in metastatic breast cancers. Exp Mol Pathol. 2016;100(3):421-5.

17. Lee JY, et al. Mutational profiling of brain metastasis from breast cancer: matched pair analysis of targeted sequencing between brain metastasis and primary breast cancer. Oncotarget. 2015;6(41):43731-42.

18. Fumagalli $\mathrm{C}$, et al., Inter-tumor genomic heterogeneity of breast cancers: comprehensive genomic profile of primary early breast cancers and relapses. Breast Cancer Research, 2020. 22(1).

19. Lee JY, et al. Gene Expression Profiling of Breast Cancer Brain Metastasis Sci Rep. 2016;6:28623.

20. Iwamoto $T$, et al. Distinct gene expression profiles between primary breast cancers and brain metastases from pair-matched samples. Sci Rep. 2019;9(1):13343. 
21. Schrijver W, et al. Mutation Profiling of Key Cancer Genes in Primary Breast Cancers and Their Distant Metastases. Cancer Res. 2018;78(12):3112-21.

22. Lehmann S, et al. Targeting p53 in vivo: a first-in-human study with p53-targeting compound APR246 in refractory hematologic malignancies and prostate cancer. J Clin Oncol. 2012;30(29):3633-9.

23. André F, et al. Alpelisib for PIK3CA-Mutated, Hormone Receptor-Positive Advanced Breast Cancer. $N$ Engl J Med. 2019;380(20):1929-40.

24. Koboldt DC, et al. Comprehensive molecular portraits of human breast tumours. Nature. 2012;490(7418):61-70.

25. Dupont Jensen J, et al. PIK3CA mutations may be discordant between primary and corresponding metastatic disease in breast cancer. Clin Cancer Res. 2011;17(4):667-77.

26. Morgan AJ, Giannoudis A, Palmieri C. The genomic landscape of breast cancer brain metastases: a systematic review. Lancet Oncol. 2021;22(1):e7-17.

27. Bareche $Y$, et al. Unravelling triple-negative breast cancer molecular heterogeneity using an integrative multiomic analysis. Ann Oncol. 2018;29(4):895-902.

28. Shah SP, et al. The clonal and mutational evolution spectrum of primary triple-negative breast cancers. Nature. 2012;486(7403):395-9.

29. Mosele, F., et al., Outcome and molecular landscape of patients with < em >PIK3CA</em>-mutated metastatic breast cancer. Annals of Oncology, 2020. 31(3): p. 377-386.

30. Baselga J, et al. Biomarker analyses in CLEOPATRA: a phase III, placebo-controlled study of pertuzumab in human epidermal growth factor receptor 2-positive, first-line metastatic breast cancer. J Clin Oncol. 2014;32(33):3753-61.

31. LoibI S, et al. PIK3CA mutations are associated with lower rates of pathologic complete response to anti-human epidermal growth factor receptor 2 (her2) therapy in primary HER2-overexpressing breast cancer. J Clin Oncol. 2014;32(29):3212-20.

32. Linderholm BK, et al. The expression of vascular endothelial growth factor correlates with mutant p53 and poor prognosis in human breast cancer. Cancer Res. 2001;61(5):2256-60.

33. Ding L, et al. Genome remodelling in a basal-like breast cancer metastasis and xenograft. Nature. 2010;464(7291):999-1005.

34. Powell E, Piwnica-Worms D, Piwnica-Worms H. Contribution of p53 to metastasis. Cancer discovery. 2014;4(4):405-14.

35. Hulsbergen AFC, et al. Subtype switching in breast cancer brain metastases: a multicenter analysis. Neurooncology. 2020;22(8):1173-81.

36. Yeung $\mathrm{C}$, et al. Estrogen, progesterone, and HER2/neu receptor discordance between primary and metastatic breast tumours-a review. Cancer Metastasis Rev. 2016;35(3):427-37.

37. Kodahl AR, et al. Correlation between circulating cell-free PIK3CA tumor DNA levels and treatment response in patients with PIK3CA-mutated metastatic breast cancer. Mol Oncol. 2018;12(6):925-35. 
38. Siravegna G, et al., Genotyping tumour DNA in cerebrospinal fluid and plasma of a HER2-positive breast cancer patient with brain metastases. ESMO Open, 2017. 2(4).

39. Keup C, et al., Longitudinal Multi-Parametric Liquid Biopsy Approach Identifies Unique Features of Circulating Tumor Cell, Extracellular Vesicle, and Cell-Free DNA Characterization for Disease Monitoring in Metastatic Breast Cancer Patients. Cells, 2021. 10(2).

\section{Tables}

Due to technical limitations, Table 1 to 5 are only available as a download in the Supplemental Files section.

\section{Figures}




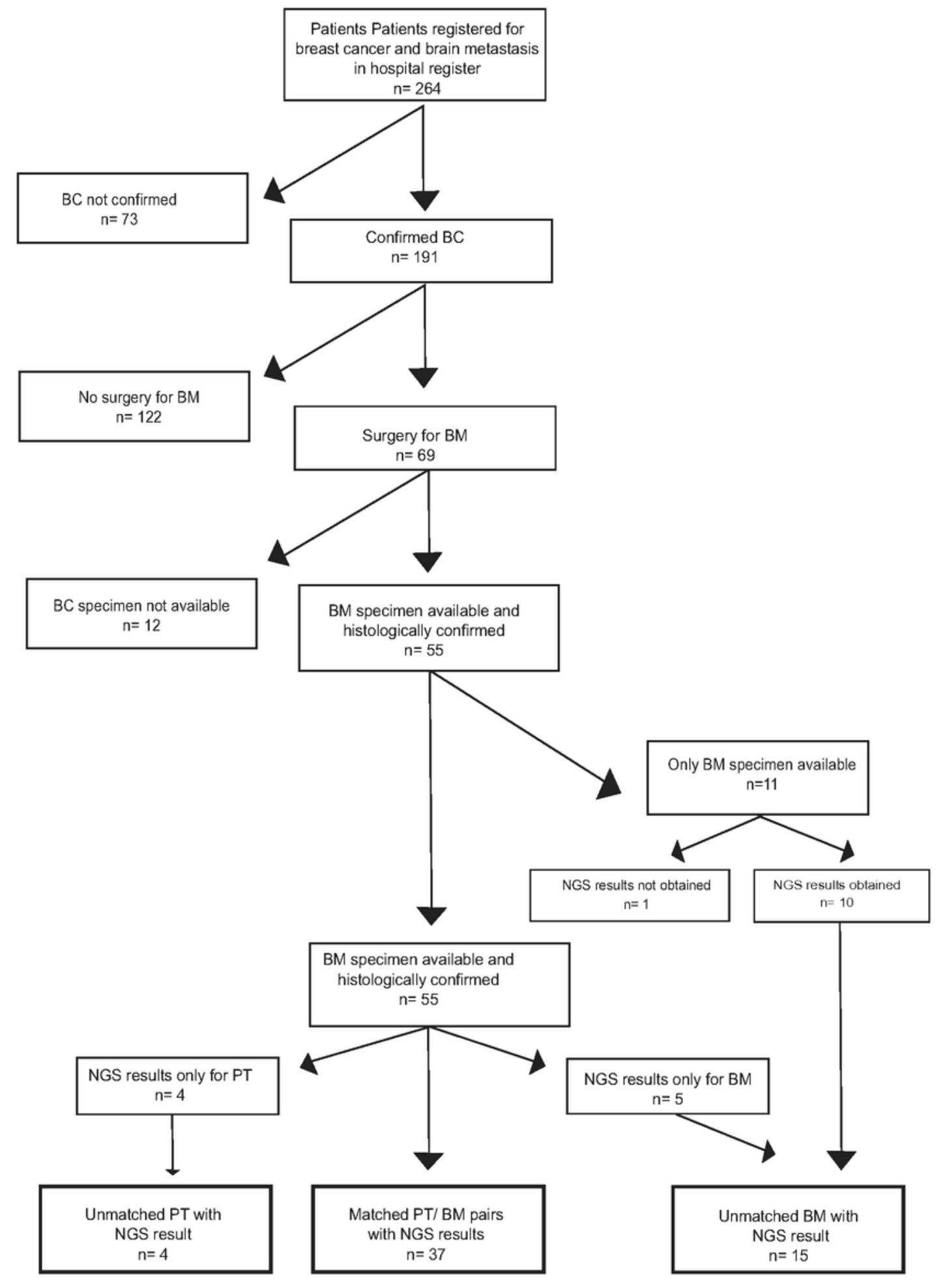

\section{Figure 1}

Consolidated Standards of Reporting Trials (CONSORT) diagram listing inclusions and exclusions and final numbers of primary tumors, brain metastases and matched primary and brain metastases. 


\begin{tabular}{|c|c|c|c|c|c|c|c|c|c|c|c|c|c|c|c|c|c|c|c|c|c|c|c|c|c|c|c|c|c|c|c|c|c|c|c|c|c|}
\hline \multirow[b]{2}{*}{ Patient ID } & \multicolumn{11}{|c|}{ Luminal } & \multicolumn{17}{|c|}{ TNBC } & \multicolumn{9}{|c|}{ HER2+ } \\
\hline & ${ }_{47}$ & 155 & 177 & 97 & 49 & 165 & 25 & 64 & 125 & 42 & 152 & $\begin{array}{l}99 \\
99\end{array}$ & 148 & 149 & 56 & 119 & 50 & 93 & 75 & 143 & 103 & 95 & 191 & 70 & 189 & 123 & 78 & 65 & 71 & 154 & 186 & 190 & 1012 & 138 & 15 & & $\begin{array}{ll}4 & 167 \\
4\end{array}$ \\
\hline Subtype & $m$ & " & & & 1 & $\mid u$ & $T_{m}$ & p. & $\mu$ & T" & & $1.1 \%$ & . & P. & p. & Pa" & p. & p. & P. & 18 & p m & P." & 1. & \%." & 7. & 1. & P." & & 4. & P. & & p. & " p & & 0. & & The \\
\hline $\begin{array}{l}\text { Brain Metastasis } \\
\text { Specific Survival } \\
\text { (months) }\end{array}$ & 57 & 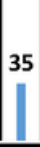 & 31 & 28 & 28 & $\begin{array}{c}19 \\
1\end{array}$ & $\begin{array}{c}15 \\
1\end{array}$ & 11 & 8 & $\begin{array}{l}7 \\
1\end{array}$ & 6 & 25 & 25 & $\begin{array}{c}18 \\
\end{array}$ & $\begin{array}{c}17 \\
17\end{array}$ & $\begin{array}{c}16 \\
16\end{array}$ & $\begin{array}{c}15 \\
15\end{array}$ & $\begin{array}{c}14 \\
11\end{array}$ & $\begin{array}{l}9 \\
1\end{array}$ & $\begin{array}{l}8 \\
1 \\
\end{array}$ & 5 & 5 & 4 & 4 & 3 & 3 & 2 & 1 & 62 & 56 & $\begin{array}{c}24 \\
1\end{array}$ & & 7 & 5 & 5 & $\underline{4}$ & 0 \\
\hline Mutation change & $\mathrm{N}$ & $N$ & A & $N$ & $\mathrm{~N}$ & $\mathrm{~N}$ & $\mathbf{L}$ & 1 & $\bar{N}$ & 1 & $\mathrm{~N}$ & $\mathrm{~N}$ & $\mathrm{~N}$ & $\mathrm{~N}$ & $\bar{N}$ & $\mathrm{~N}$ & 1 & $\mathrm{~N}$ & $\mathrm{~N}$ & $\mathrm{~N}$ & $\mathrm{~N}$ & $\mathrm{~N}$ & 1 & 10 & N & $N$ & $\mathrm{~N}$ & 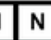 & L & $\mathrm{N}$ & $\mathbf{L}$ & $\mathrm{N}$ & 1 & $\mathrm{~N}$ & $\bar{N}$ & $\bar{N}$ & $\mathrm{~N}$ \\
\hline TP53 & & & & & & & & & & & & & & & & & & & & & & & & & & & & & & & & & & & & & \\
\hline PIK3CA & & & & & & & & & & & & & & & & & & & & & & & & & & & & & & & & & & & & & \\
\hline$\overline{\mathrm{ABL} 1}$ & & & & & & & & & & & & & & & & & & & & & & & & & & & & & & & & & & & & & \\
\hline $\mathrm{CDH1}$ & & & & & & & & & & & & & & & & & & & & & & & & & & & & & & & & & & & & 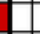 & \\
\hline$\overline{C D K N 2 A}$ & & & & & & & & & & & & & & & & & & & & & & & & & & & & & & & & & & & & & \\
\hline EGFR & & & & & & & & & & & & & & & & & & & & & & & & & & & & & & & & & & & & & \\
\hline ERBB4 & & & & & & & & & & & & & & & & & & & & & & & & & & & & & & 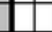 & & & & & & & \\
\hline HRAS & & & & & & & & & & & & & & & & & & & & & & & & & & & & & & & & & & & & & \\
\hline MET & & & & & & & & & & & & & & & & & & & & & & & & & & & & & & $=$ & & & & & & & \\
\hline PTEN & & & & & & & & & & & & & & & & & & & & & & & & & & & & & & 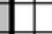 & & & & & & & \\
\hline PTPN11 & & & & & & & & & & & & & & & & & & & & & & & & & & & & & & & & & & & & & \\
\hline RB1 & & & & & & & & & & & & & & & & & & & & & & & & & & & & & & & & & & & & & \\
\hline
\end{tabular}

\begin{tabular}{|l|l|}
\hline Luminal A \\
\hline Luminal B \\
\hline TNBC \\
\hline HER2+/ER+ \\
\hline HER2+/ER. \\
\hline
\end{tabular}

Pathogenic mutation

Uncertain pathogenicity

\begin{tabular}{|l|l|l}
\hline Uncertain in primary, uncertain and pathogenic in BM & *
\end{tabular}

\section{Figure 2}

All the matched pairs of primary and brain metastatic tumors color coded in to sub groups with pathological and uncertain mutations shown as well as brain metastasis specific survival. 


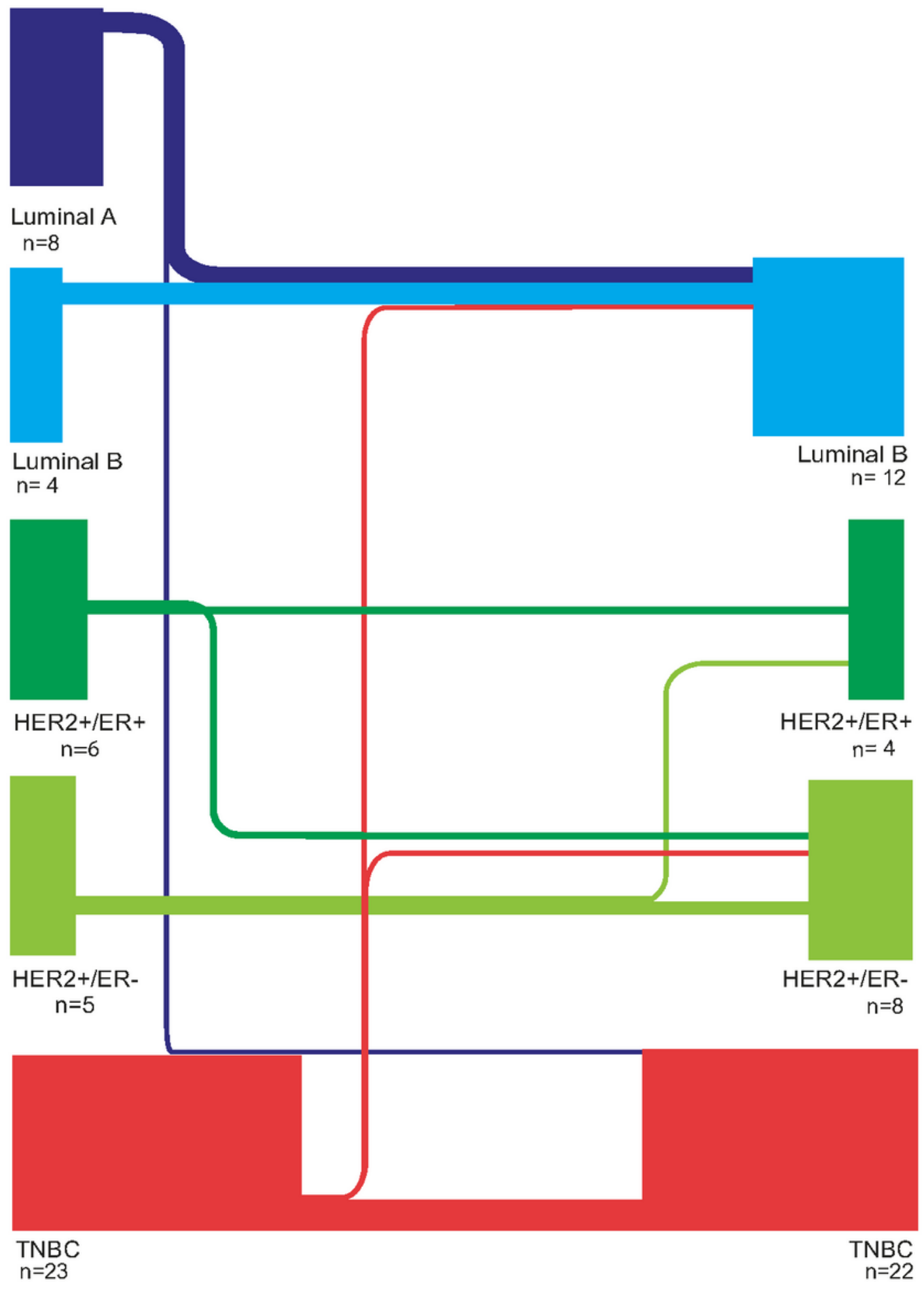

\section{Figure 3}

Histological expression in primary tumors and brain metastases, divided in to groups to depict the number of tumors that had significantly changed receptors so that the subgroup was altered. The thickness of the line represents the number of altered in each group.

\section{Supplementary Files}


This is a list of supplementary files associated with this preprint. Click to download.

- SupplementalData.xlsx

- Tables.docx 